\title{
MIMO SVD-BASED MULTIPLEXING WITH IMPERFECT CHANNEL KNOWLEDGE
}

\author{
Michael D. Larsen* \\ Brigham Young University \\ Provo, Utah 84606
}

\author{
A. Lee Swindlehurst \\ University of California, Irvine \\ Irvine, California 92697
}

\begin{abstract}
In narrowband multiple-input multiple-output (MIMO) communication systems with perfectly known channel state information (CSI), the singular value decomposition (SVD) is commonly used to decompose the MIMO channel into independent single-input single-output subchannels. In theory, optimal interference-free data multiplexing may then be carried out using the subchannel power levels provided by the well-known waterfilling solution. In practice, however, when finite codebooks are used and perfect CSI is unavailable, adaptations to power levels and bit-loading schemes are often needed to maintain reasonable performance. In this paper, we use expressions for the per-subchannel signal-to-interference-and-noise ratio for the imperfect CSI case to derive approximately optimal subchannel power levels and thresholds on the amount of CSI imperfections and noise tolerable in SVD-based multiplexing systems using $M$-ary quadrature amplitude modulation. Numerical simulations demonstrate the usefulness of the derived expressions.
\end{abstract}

Index Terms - MIMO systems, Singular value decomposition, Resource management, Adaptive modulation

\section{INTRODUCTION}

In narrowband multiple-input multiple-output (MIMO) communication systems with perfectly known channel state information (CSI), the singular value decomposition (SVD) may be used to decompose the MIMO channel into multiple independent single-input single-output channels, enabling interference-free data multiplexing. MIMO SVD-based methods include the well-known waterfilling solution which maximizes information throughput over the MIMO channel given a fixed transmission power [1,2]. Given subchannel power levels, such as those provided by the waterfilling solution, the signal-to-noise ratios of the SVD subchannels may be used to devise bit-loading and coding schemes that approach the available channel capacity.

Unfortunately, it is not possible in practice to obtain perfect CSI since realizable channel estimates are formed from

* This work was supported by the U.S. Army Research Office under the Multi-University Research Initiative (MURI) grant W911NF-04-1-0224 and by the National Science Foundation under grant CCF-0313056. noisy measurements and system mobility results in rapidly changing channel conditions. When noisy or outdated CSI is used in conjunction with an SVD-based multiplexing method, the MIMO subchannels become coupled, resulting in potentially severe subchannel power loss and interference. As a consequence, subchannel bit-loading levels selected using the signal and noise powers of the perfect CSI assumption may no longer meet required probability-of-error levels. Some of the effects of imperfect CSI (ICSI) on MIMO SVD-based multiplexing methods that employ common signal constellations (such as $M$-ary quadrature amplitude modulation, or MQAM) have been examined in [3-5], and ad-hoc subchannel power and bit-loading methods have been proposed in $[4,5]$ to compensate for the effects of ICSI and finite symbol constellations.

In this paper, we present a more deterministic design approach that uses the per-subchannel SINR expression derived in [6] (also see [5]) to find a subchannel power-loading scheme and multiplexing thresholds for SVD-based MIMO signaling with M-QAM constellations under ICSI conditions. In particular, the SINR expression is used to derive approximately optimal subchannel power levels for M-QAM signaling under a subchannel SER constraint. Using these power levels, thresholds are found for the amount of channel uncertainty and measurement noise tolerable when multiplexing over the SVD-based subchannels. These results allow for the design of bit-loading schemes with improved performance and robustness relative to waterfilling when perfect CSI is unavailable. Note that a more complete exposition of this topic may be found in [6].

The remainder of this paper will proceed as follows. In Section 2, the MIMO transmission model and the ICSI SINR expression are presented. These results are then used in Sections 3 and 4 to find the optimal subchannel power levels and multiplexing thresholds, respectively. Finally, numerical simulations in Section 5 are used to demonstrate the accuracy of the derived expressions and their usefulness when applied to the MIMO SVD-based bit-loading problem.

\section{SIGNAL MODEL}

Consider a narrow-band flat-fading MIMO scenario in which CSI estimated at the receiver is fed back to the transmitter 
over an error-free channel. As such, both the transmitter and receiver have identical estimates of the MIMO channel. We assume the following SVD-based signaling scheme:

$$
\mathbf{y}=\mathbf{U}^{H}[(\mathbf{H}+\Delta \mathbf{H}) \mathbf{V P x}+\mathbf{n}],
$$

where $\mathbf{n}$ represents zero-mean Gaussian noise, $\mathbf{H}$ is the $N_{r} \times$ $N_{t}$ MIMO channel matrix as known at the transmitter and receiver, and $\mathbf{H}+\Delta \mathbf{H}$ is the actual MIMO channel encountered during transmission. The matrix $\mathbf{H}$ has the following (compact) singular value decomposition (SVD):

$$
\mathbf{H}=\mathbf{U} \boldsymbol{\Sigma} \mathbf{V}^{H},
$$

where $\mathbf{U}$ and $\mathbf{V}$ are respectively $N_{r} \times F$ and $N_{t} \times F, \boldsymbol{\Sigma}=$ $\operatorname{diag}\left\{\sigma_{1}, \sigma_{2}, \cdots, \sigma_{F}\right\}, F=\min \left\{N_{r}, N_{t}\right\}$ with the singular values in order of decreasing size, and $(\cdot)^{H}$ denotes the Hermitian tranpose. The matrix $\mathbf{P}$ is a real-valued $F \times F$ diagonal power-loading matrix whose entries determine the signal power assigned to the unit-variance elements of the signal vector $\mathbf{x}$. The diagonal entries of $\mathbf{P}$ are constrained so that the average total transmit power is equal to some total transmit power $P_{T}$, i.e., $\operatorname{tr}\left(\mathbf{P}^{2}\right)=P_{T}$.

Assume now that the signal vector $\mathbf{x}$, the channel perturbation $\Delta \mathbf{H}$, and the measurement noise $\mathbf{n}$ are mutually independent ${ }^{1}$; that the elements of $\mathbf{x}$ are zero mean and uncorrelated with unity variance; and that the elements of $\Delta \mathbf{H}$ are zero-mean complex circular-symmetric Gaussian distributed $\operatorname{vec}(\Delta \mathbf{H}) \sim \mathcal{C N}\left(\mathbf{0}, \sigma_{H}^{2} \mathbf{I}\right)$, where the vectorization operator $\operatorname{vec}(\mathbf{A})$ stacks the columns of the matrix $\mathbf{A}$. Under these assumptions, the average per-subchannel SINR for the $i$-th subchannel is given by [6]

$$
\operatorname{SINR}_{i}=\frac{\left(\sigma_{i}^{2}+\sigma_{H}^{2}\right)[\mathbf{P}]_{i i}^{2}}{\sigma_{H}^{2}\left(P_{T}-[\mathbf{P}]_{i i}^{2}\right)+\sigma_{n}^{2}} .
$$

\section{SUBCHANNEL POWER ALLOCATION}

Consider a communications system in which the transmission over each subchannel is required to satisfy a symbol error rate (SER) threshold, $\mathcal{E}$. When only one subchannel is in use, the scheme is referred to as beamforming; on the other hand, transmission over multiple subchannels results in spatial multiplexing. Using the SINR expression given above, we wish to determine the best way to allocate power to the MIMO subchannels in order to maximize the resulting transmission rate; this includes determining when it is best to abandon multiplexing in favor of beamforming.

In order to proceed, we must first relate the SINR, the number of bits per transmission symbol, and the SER. This is done for M-QAM signals using two approximations. The first relates the SER to the SINR and the minimum distance

\footnotetext{
${ }^{1}$ Although noise is partially responsible for the CSI errors, we assume that the current realization $\mathbf{n}$ of the temporally white noise was not used in calculating the receiver CSI.
}

between constellation points for a constellation with unit average energy [7]:

$$
\mathrm{SER} \approx 4 Q\left(\sqrt{d_{m i n}^{2} \mathrm{SINR}}\right)
$$

where $Q$ is the well-known Q-function for Gaussian distributions and $d_{\min }$ is the minimum constellation distance. The second relates the minimum distance between M-QAM constellation points to bits per M-QAM symbol by fitting the following first-order-in-the-exponent curve to the constellation points:

$$
d_{\text {min }}^{2}=e^{-\alpha b+\beta}
$$

where $b$ is the number of bits per symbol. Assuming symmetric constellations, a least squares fit of $\alpha$ and $\beta$ to (5) results in parameter values $\alpha=0.717, \beta=0.604$. Using (4) and (5), we may then approximate the number of bits per transmission as a function of the SER and SINR:

$$
b \approx \frac{1}{\alpha}\left(\beta-\ln \left(\frac{\left(Q^{-1}(\mathrm{SER} / 4)\right)^{2}}{\mathrm{SINR}}\right)\right)
$$

where $Q^{-1}(\cdot)$ is the inverse Q-function. Note that any suboptimality in the approximately optimal power allocation developed next is a result of the approximations in (6).

The expressions in (3) and (6) allow for a systematic optimization of the subchannel power levels. To that end, consider the following constrained optimization problem, where for a given number of active subchannels $M \leq F$, a diagonal power loading matrix $\mathbf{P}$ with positive, real-valued elements is chosen to maximize the number of M-QAM bits per transmission while guaranteeing a minimum $\mathrm{SER}$ of $\mathcal{E}$ :

$$
\mathbf{P}^{*}=\arg \max _{\substack{\mathbf{P}: \sum[\mathbf{P}]_{f f}^{2}=P_{T} \\ \operatorname{SER} \geq \mathcal{E}}} \sum_{f=1}^{M} b_{f} .
$$

This general optimization problem is difficult to solve, but the approximation obtained by substituting (6) into (7) can be solved in closed form. This new optimization problem may be solved using Lagrange multipliers by minimizing the cost function

$$
J=\sum_{f=1}^{M} \ln \left(\frac{P_{I N, f}}{P_{s, f}}\right)-\gamma\left(\sum_{f=1}^{M}[\mathbf{P}]_{f f}^{2}-P_{T}\right) .
$$

Equating the derivative of $J$ with respect to $[\mathbf{P}]_{i i}^{2}$ to zero, we find after some manipulation and reasoning that the optimal power loading scheme when using $M \leq F$ active subchannels is to spread the power uniformly over the subchannels, i.e.,

$$
[\mathbf{P}]_{i i}^{2 *}=\frac{P_{T}}{M} .
$$

Note that this solution always corresponds to a minimum of the cost function (second derivative is always positive). The 
power level in (9) is for a particular $M \leq F$. The optimal $M$ may be selected as the value of $M$ which results in the largest value for

$$
J_{2}=\sum_{f=1}^{M} \ln \left(\frac{P_{s, f}}{P_{I N, f}}\right)
$$

with the power allocated evenly over the $M$ active subchannels. Since $F$ is typically not large, the cost of this search is small.

\section{BEAMFORMING VS. MULTIPLEXING THRESHOLDS}

Assuming uniform power allocation as discussed above, we now derive thresholds for the CSI error and noise level above which beamforming rather than spatial multiplexing is optimal in terms of bits per transmission. We say multiplexing over $M$ subchannels is preferable to beamforming in terms of bits per transmission when

$$
b_{B F} \leq \sum_{m=1}^{M} b_{m}
$$

where $b_{B F}$ is the number of bits supported when beamforming and $b_{1}, b_{2}, \ldots, b_{M}$ are the number of bits supported when multiplexing using the first $M$ subchannels (ordered by associated singular values). If we assume that $\sigma_{m}^{2} \gg \sigma_{H}^{2}$ near the threshold for the $M$ active subchannels, we may use (6), the SINR expression of (3), and the uniform power allocation assumption to obtain the threshold

$\sigma_{H, M}^{2 *}=\frac{\left[\left(\frac{P_{T} e^{\beta}}{\left(Q^{-1}(\mathcal{E} / 4)\right)^{2}}\right)^{M-1}\left(\prod_{m=2}^{M} \sigma_{m}^{2}\right) \sigma_{n}^{2}\right]^{\frac{1}{M}}-M \sigma_{n}^{2}}{(M-1) P_{T}}$

where multiplexing is optimal for $\sigma_{H}^{2} \leq \sigma_{H, M}^{2 *}$ and beamforming is optimal otherwise [6]. The above threshold describes the optimal transition point between multiplexing over $M$ subchannels and beamforming. An overall threshold may be found by evaluating (12) for possible values of $M$ and selecting the maximum, i.e,

$$
\sigma_{H}^{2 *}=\max _{M} \sigma_{H, M}^{2 *}
$$

Once again, we note that since $F$ is typically not large, the cost of this search is small.

Note that the threshold of (13) must be positive in order for multiplexing to be useful for any value of $\sigma_{H, 2}^{2}$. Using this observation, we obtain the following threshold on the measurement noise above which beamforming is always preferable:

$$
\sigma_{n}^{2 *}=\left(\frac{P_{T} e^{\beta}}{M^{*}\left(Q^{-1}(\mathcal{E} / 4)\right)^{2}}\right)\left(\frac{\prod_{m=2}^{M^{*}} \sigma_{m}^{2}}{M^{*}}\right)^{\frac{1}{M^{*}-1}}
$$

where $M^{*}$ is the maximizing $M$ from (13).

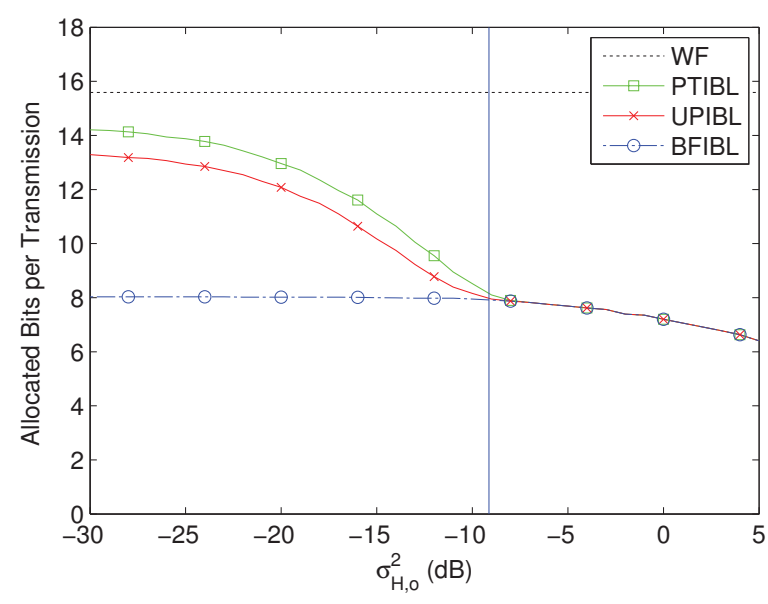

Fig. 1. A comparison of designed bit-loading levels for uninformed and informed bit-loading schemes.

\section{NUMERICAL SIMULATIONS}

We now examine the performance of the uniform subchannel power MIMO-SVD multiplexing scheme, which we will refer to as uniform power with informed bit loading (UPIBL), and the multiplexing/beamforming threshold via numerical simulation. For the simulations, assume a $4 \times 4$ MIMO channel with $\mathbf{H}$ and $\Delta \mathbf{H}$ selected such that $\operatorname{vec}(\mathbf{H}) \sim \mathbb{C N}\left(\mathbf{0}, \mathbf{I}_{16}\right)$ and $\operatorname{vec}(\Delta \mathbf{H}) \sim \mathbb{C} \mathcal{N}\left(\mathbf{0}, \sigma_{H}^{2} \mathbf{I}_{16}\right)$. To prevent channel power growth, the resulting channel $\mathbf{H}+\Delta \mathbf{H}$ is normalized. The channel perturbation is assumed to be a result of both measurement noise (see [8]) and mobility (see [9] relative to the Gauss-Markov model). The signal power and noise variance are $P_{T}=1$ and $\sigma_{n}^{2}=-20 \mathrm{~dB}$, respectively, at each receive antenna. The target SER is $\mathcal{E}=0.01$, and outages are said to occur when the instantaneous SER exceeds this level. The results of the simulations are averaged over 100 channel realizations with 100 Monte Carlo perturbation trials per channel realization.

In the simulations, UPIBL will be compared with three other methods: conventional waterfilling (WF), beamforming with informed bit-loading (BFIBL), and the ad-hoc method of [5] which we refer to as power trimming with informed bit loading (PTIBL). In this iterative method, waterfilling subchannel power levels are trimmed so that $\mathcal{E}$ is met as closely as possible, and excess subchannel power is reallocated in an effort to ensure that all available power is directed towards an increase in throughput.

Figure 1 displays the sum of the bits allocated to each subchannel by the various methods versus the channel perturbation variance. Since the channel perturbation variance due to CSI estimation error has a fixed effect for a given noise variance, the results are plotted relative to $\sigma_{H, o}^{2}$, the perturbation variance due to mobility alone. WF allocates 


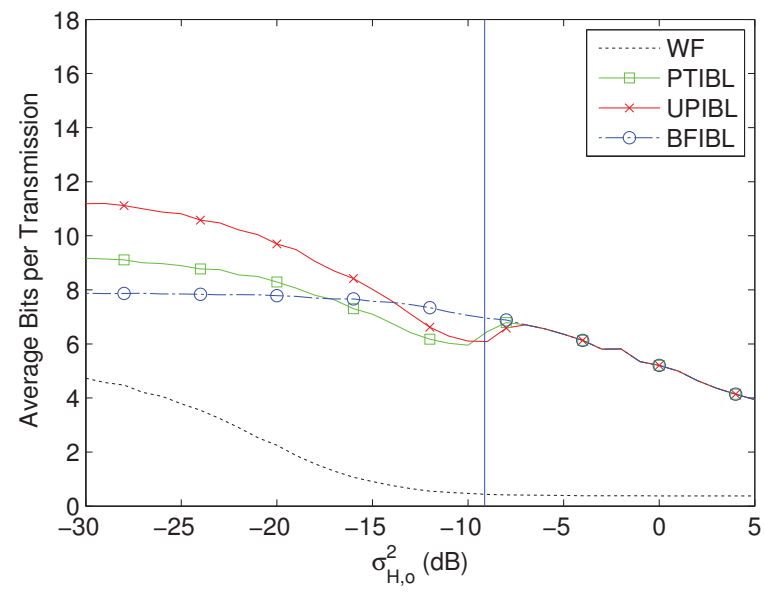

Fig. 2. A comparison of the average bits per transmission achieved using the uninformed and informed bit-loading schemes when accounting for subchannel outages.

the same number of bits per transmission (BPT) regardless of $\sigma_{H}^{2}$ since it does not take CSI errors into account. Of the informed methods, the PTIBL method allocates the most BPT, and it remains superior or equivalent in this regard to the other methods over the entire range of considered channel perturbation powers. This performance advantage is expected due to the power trimming, which assures all available power is directed towards bit allocation. For this reason, in the absence of measurement-noise-induced channel perturbations, PTIBL will also allocate more bits than WF for low channel perturbation variances, though we don't show that scenario here. Interestingly, in the measurement-noise-free scenario, the UPIBL method also allocates slightly more BPT than WF at low channel perturbation levels, since unlike UPIBL, WF is not optimized for the case of finite M-QAM constellations.

Allocating largest number of bits, however, does not necessarily result in the best throughput after outages are considered. In Fig. 2, we plot the average effective transmission rates, assuming that bits corresponding to SER outages are lost. Of the informed methods, PTIBL, in particular, has a poor outage performance at low channel perturbation levels. This is a result of its power trimming, which leaves no power margin to protect its transmissions against outages. Consequently, UPIBL ends up having superior performance relative to PTIBL in terms of actual throughput.

The vertical lines in Figs. 1 and 2 indicate the location of the channel perturbation threshold $\sigma_{H}^{2 *}$. In Fig. 1, we see that the threshold accurately determines the point at which the bit allocation switches from multiplexing to beamforming (the point at which the UPIBL allocation equals that of BFIBL). When outages are taken into account, the threshold is optimistically high. However, this problem may be countered by modifying the SINR expressions to reduce the overall number of outages, as discussed in [6].

In summary, we note that using SINR expressions to aid power and bit allocation design in MIMO-SVD systems appears to be effective in addressing the effects of interference resulting from imperfect CSI. Also, it is particularly interesting to observe that, while simple, UPIBL outperforms the more adaptive and computationally intensive PTIBL method when including the effect of outages. As mentioned previously, much of this performance advantage is a result of the excess power UPIBL leaves on the subchannels, the very excess PTIBL attempts to remove. It is also interesting to consider that the power reallocation performed in PTIBL may actually tend to push this method closer, at least on average, to a uniform power allocation.

\section{REFERENCES}

[1] I. E. Telatar, "Capacity of multi-antenna gaussian channels,” AT\&T Bell Labs, Internal Tech. Memo., Jun. 1995.

[2] G. J. Foschini and M. J. Gans, "On limits of wireless communications in a fading environment when using multiple antennas," Wireless Pers. Commun., vol. 6, pp. 311-335, Mar. 1998.

[3] Z. Zhou, B. Vucetic, M. Dohler, and Y. Li, "MIMO systems with adaptive modulation," IEEE Trans. Veh. Technol., vol. 54, pp. 1828-1842, Sep. 2005.

[4] X. Zhang and B. Ottersten, "Power allocation and bit loading for spatial multiplexing in MIMO systems," in Proc. ICASSP, Honolulu, Hawaii, Apr. 2003, pp. V 5356.

[5] H. Zhang, D. L. Goeckel, and G. Ananthaswamy, "Adaptive modulation in MIMO eigenbeamforming with outdated channel state information," in Proc. GLOBECOM, San Fransisco, CA, USA, Nov. 2006, pp. 1-5.

[6] M. Larsen and A. L. Swindlehurst, "Power allocation and bit loading for spatial multiplexing with imperfect CSI," IEEE Trans. Signal Process., submitted for publication.

[7] J. G. Proakis, Digital Communications, 4th ed. New York, NY: McGraw-Hill, 2001.

[8] T. Svantesson and A. L. Swindlehurst, "A performance bound for prediction of MIMO channels," IEEE Trans. Signal Process., vol. 54, pp. 520-529, Feb. 2006.

[9] C. B. Peel and A. L. Swindlehurst, "Performance of space-time modulation for a generalized time-varying rician channel model," IEEE Trans. Wireless Commun., vol. 3, pp. 1003-1012, May 2004. 